\title{
An evaluation of general practitioners' experience of LactaMap, an online lactation care support system
}

Melinda Boss, Jennifer Turner, Tracey Hirst, Douglas Pritchard, Rafael Pérez-Escamilla, Rhonda Clifford

\section{Background and objective LactaMap is an online lactation care support system designed to assist general practitioners (GPs) caring for breastfeeding women and infants. The aim of this study was to qualitatively evaluate GPs' experience of the prototype LactaMap website.}

\section{Methods}

The study was conducted in Perth, Western Australia, with five GPs in 2018/2019 by integrating a Think Aloud protocol and a semi-structured interview. Data were transcribed and uploaded to ATLAS.ti 8 Qualitative Data Analysis Software. Descriptive coding was themed deductively and analysed.

\section{Results}

All participants responded positively to the LactaMap website and indicated that it was valuable. A small number of critical usability issues were identified.

\section{Discussion}

Integrating two qualitative approaches provided information about what may influence LactaMap's adoption by GPs. The integrated data allowed evaluation of LactaMap as it is and also considered what might be possible, facilitating a customised lactation decision support tool for general practice.
GLOBALLY, BREASTFEEDING RATES FALL well short of World Health Organization recommendations of exclusive breastfeeding for six months, with continued breastfeeding during the introduction of complementary foods until at least two years of age. The prevalence of breastfeeding at 12 months is lower than $20 \%$ in most high-income countries. ${ }^{1}$ Breastfeeding durations are longer in lowand middle-income countries, but even in these settings only $37 \%$ of infants younger than six months of age are exclusively breastfed. ${ }^{1}$ The health, human capital and economic costs of early weaning for children, mothers and society are significant. ${ }^{2}$ Nutrition-related shortfalls in developmental potential affect schooling and adult income. Together these make up the largest component of economic losses, estimated to total more

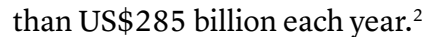

General practitioners (GPs) are frequently consulted by mothers and babies in the first six months postpartum and have been shown to significantly influence their patients' infantfeeding decisions. ${ }^{3-5}$ When addressing breastfeeding challenges, GPs report that they have not received the lactation education needed for the knowledge and skills expected of them. ${ }^{6-8}$ Insufficient time and poor accessibility of evidence have been identified as impediments to their ability to make use of research data. ${ }^{9}$ To deliver this knowledge in an accessible format, a multidisciplinary group from
The University of Western Australia developed LactaMap, an online lactation care support system..$^{10}$ LactaMap aims to support GPs caring for women and infants requiring lactation support by delivering evidence-based lactation information in an accessible format at the point of care. The comprehensive website includes over 100 evidence-based clinical practice guidelines, supporting information and patient information documents, all underpinned by more than 1000 references.

The everyday use of digital services has become the norm in health. ${ }^{11}$ Online clinical decision support systems have the potential to improve healthcare by delivering evidence-based clinical information at the point of care and encouraging the use of practice guidelines. ${ }^{12}$ In order to facilitate adoption and uptake, LactaMap is designed to be used intuitively and without prior training. ${ }^{13}$

Qualitative research methods are particularly useful to test the feasibility and utility of health interventions in the real-world context for which they were designed. ${ }^{14,15}$ Testing during development provides important preparatory information to evaluate acceptability and improve design prior to implementation. ${ }^{16,17}$ User experience is a key factor influencing adoption and use of online decision support systems by doctors. ${ }^{13}$ Testing the user's experience with a website provides information about how easy or pleasing it is to use and detect 
areas of the user interface that work well or need improvement. ${ }^{18}$ This can then be used to guide customisation to specifically meet the unique requirements of general practice. The aim of this study was to evaluate GPs' user experience of the prototype LactaMap website by integrating the results of two qualitative methods.

\section{Methods}

\section{Intervention description}

The target users of LactaMap are GPs. The clinical condition targeted is lactation, and the patient population consists of mothers and term infants aged from birth to two years of age. The context for use is at the time-limited point of care (during a medical consultation with a breastfeeding mother and/or infant). LactaMap content is therefore intentionally succinct and intended to be easily navigated. Links to additional supporting information and to PubMed for references cited are included if more information is desired.

\section{Participants and procedures}

A purposive sample of GPs from the Perth metropolitan area in Western Australia were invited by a research associate (JT) to participate during September-October 2018. GP users nominated their preferred location for data collection (home, clinic or university campus). Interviews were conducted in person with the research associate, who trained by piloting both protocols with researchers experienced in qualitative research and health professionals with clinical experience.

After providing written consent to participate, GPs were required to register with the LactaMap website and use one of three case scenarios to guide navigation around the website. They were instructed to imagine themselves in consultation with the patient. This was followed by a semi-structured interview, which included a self-reported rating of confidence in treating patients with breastfeeding issues. This was rated on a Likert scale from 1 (not confident at all) to 5 (very confident). Participants received a $\$ 100$ gift voucher as compensation for time taken to complete the case scenario and interview.

\section{User feedback processes}

User experience evaluation of the

LactaMap website was conducted by integrating two complementary data-gathering techniques. An objective task-oriented evaluation was carried out using the Think Aloud protocol, and a subjective evaluation was conducted via a semi-structured interview. The Think Aloud protocol is a cognitive analysis method that requires users to verbalise their thoughts as they move through a user interface towards task completion. ${ }^{19}$ A sign with the text 'please keep talking' was attached to the computer monitor, and GP users were also prompted with these words verbally if they were silent for more than 10 seconds. This technique, with limited prompts, draws on simple working memory without introducing mental processing that can influence the focus of attention. ${ }^{20}$ Almost all of the user's conscious effort is focused on task completion, with little room for reflection, feelings or opinions. ${ }^{21}$ Once this task was accomplished, each GP user completed a semi-structured interview that included a set of open-ended questions. ${ }^{22}$ This allowed broader exploration of the individual's subjective views about the softwaresupported intervention, including whether it contained the information they needed, its potential usefulness for a consultation involving breastfeeding and whether they would be likely to use it for decision support, with reasons discussed for given responses.

To mimic expected use of the LactaMap website, each GP user was asked to use the allocated case to guide navigation through website content until a decision point was reached. Each case scenario represented a different presentation of a lactation condition, and each one varied in clinical complexity. The different scenarios were intended to capture the widest possible use of LactaMap. Lactation terms used across case studies were consistent with terminology recommended in LactaPedia. ${ }^{23}$ Case scenario 1 was related to mastitis, a common condition that is diagnosed in $20 \%$ of lactating women. ${ }^{24}$ As a result of its common presentation during lactation, GP users were expected to be familiar with this diagnosis, its assessment and management. Case scenario 2 was related to maternal concerns regarding low milk synthesis. Maternal perception of low milk synthesis has been reported to be the main reason for breastfeeding cessation in 35\% of women that wean early. ${ }^{25}$ Although relatively common, approaches for management are more complex, particularly in the absence of standard objective tests to assess milk production. ${ }^{26}$ Case scenario 3 related to the breastfeeding challenges associated with an infant diagnosed with tracheomalacia, a condition characterised by insufficient supporting cartilages of the trachea. ${ }^{27}$ This was chosen because it is a rare presentation, with unknown incidence and prevalence, and therefore one with which GP users would be likely to have less experience. ${ }^{27}$ All case scenarios were reviewed by an experienced GP (DP) for appropriateness of clinical content. Each GP user was assigned one of the case scenarios based on rotation, rather than cases being individually randomised. GP user 1 received case scenario 1, GP user 2 received case scenario 2 and GP user 3 received case scenario 3; GP user 4 then received case scenario 1 again and so on. Users were informed that there was no 'right' or 'wrong' way to use the website and that their clinical decisions were not being assessed. The ability of users to locate the information they needed to support care relating to the particular case scenario they were presented (decision point) was the task under investigation for usability assessment.

\section{Data analysis}

Both the Think Aloud protocol and the semi-structured interview were recorded using an Olympus DM-3 digital voice recorder and audio transcribed verbatim. Transcripts were uploaded to ATLAS. ti 8 Qualitative Data Analysis Software for Mac to facilitate coding. Coding was conducted by identifying quotes of relevance to issues with LactaMap usability and then grouping these into categories. This descriptive coding of transcriptions and grouping into categories was the data analysis protocol. The categories were then themed deductively on the basis of attributes related to seven facets of user 
experience (Table 1)..$^{28}$ Each code was also given a usability ranking that related to its effect on task completion, from critical to positive (Table 1 ). This was a modified version of a ranking system used in previous studies. ${ }^{19,29}$ Coding was conducted independently by two study authors (MB and JT), then results were compared and discussed until consensus was reached. This process was repeated for usability ranking and theming. Thematic saturation was defined as the point at which no new categories emerged under each of the themes. ${ }^{30}$

Ethical approval to conduct the study, reference number RA/4/20/4284, was granted by The University of Western Australia Human Ethics Research Committee.

\section{Results}

A total of six GP users were interviewed, with thematic saturation reached after participant 4. Participant details are described in Table 2. Time taken to complete the Think Aloud protocol plus semi-structured interview ranged from 35 to 50 minutes.

\section{Usability testing}

Figure 1 shows user feedback regarding the features of the prototype LactaMap website. The usability rankings were visually illustrated using a coloured scale. Of 358 coded quotes, $222(62 \%)$ related to an aspect the user liked about the website, and 30 (8\%) related to critical issues that prevented task completion.

\section{User experience}

User experience was described according to seven themes derived from Moreville's model for user experience design. ${ }^{28}$

\section{Accessible}

In this study, the accessibility theme related to whether users considered the website and its content to be something they would be likely to access in the context of time-limited medical consults. Most users considered the website and its content accessible:

Something like LactaMap, which tries to give you information that is useful right then, comes in very handy because it's a lot better than saying to people, 'I'm going to have a think and go away and come back tomorrow'. [Participant (P) 2]

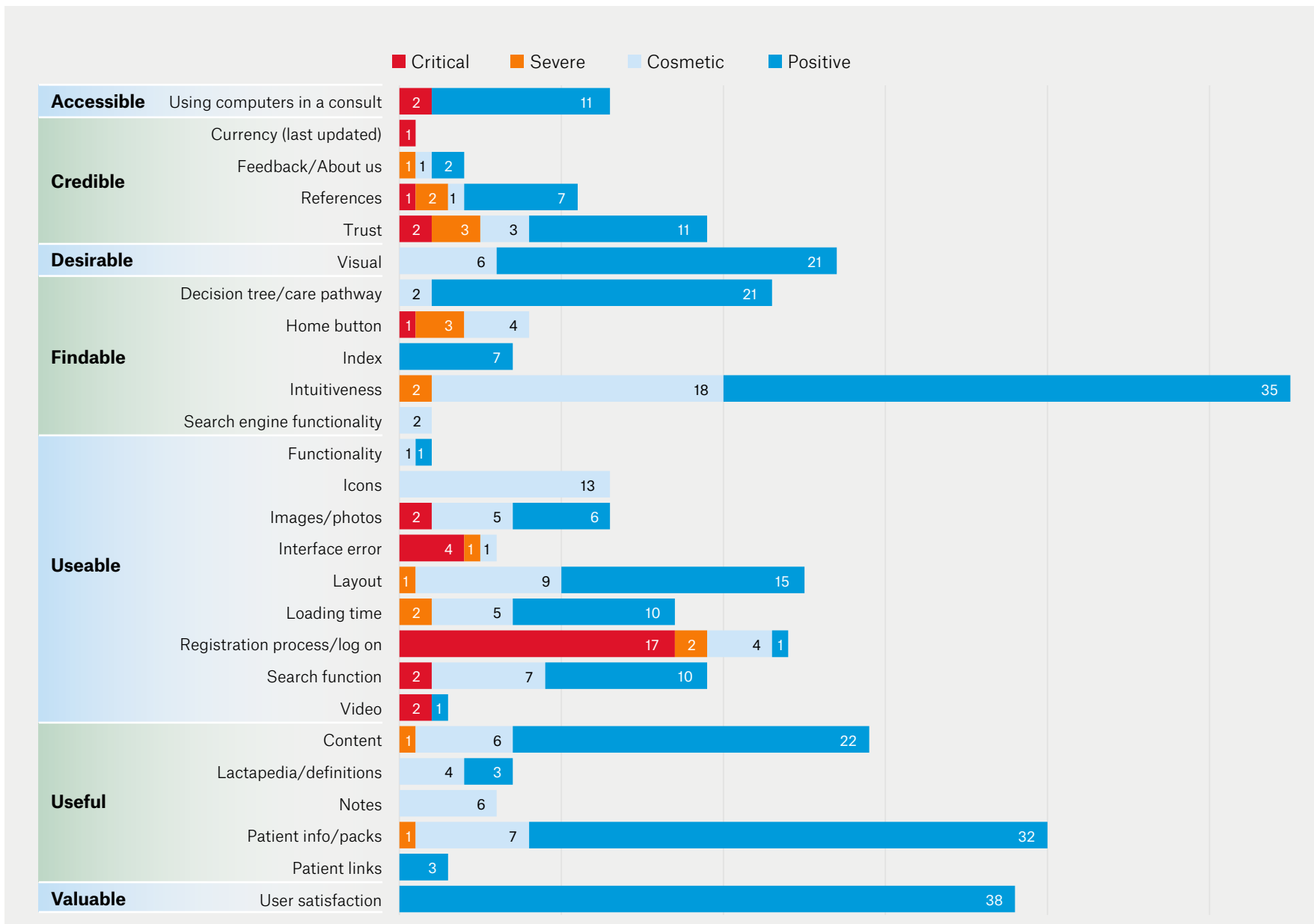

Figure 1. Themes, codes and their usability ranking (combined data from the Think Aloud protocol and semi-structured interviews) 


\section{Table 1. Facets of user experience and usability rankings with definitions ${ }^{19,28,29}$}

\begin{tabular}{ll}
\hline Facet of user experience & Definition \\
\hline Accessible & $\begin{array}{l}\text { User's ability to access the website and its content } \\
\text { during consults }\end{array}$ \\
\hline Credible & Users trust and believe what the website tells them \\
\hline Useful & Website is of practical value to the user \\
\hline Desirable & Design elements evoke emotion and appreciation \\
\hline Findable & Content is navigable and locatable \\
\hline Useable & $\begin{array}{l}\text { Users can effectively and efficiently achieve their } \\
\text { end objective }\end{array}$ \\
\hline Valuable & $\begin{array}{l}\text { User satisfaction with the website and belief that it } \\
\text { delivers value }\end{array}$ \\
\hline Usability ranking & Definition \\
\hline Critical & Unable to complete task \\
\hline Severe & Significant delay or frustration in task completion \\
\hline Cosmetic & Minor issue that may or may not affect task completion \\
\hline Positive & Something the user liked about the website \\
\hline
\end{tabular}

Two users noted that the login process might be a problem if it took too long or required them to remember 'yet another username and password' [P2].

\section{Credible}

This theme related to whether users found the website trustworthy. All but one commented that the inclusion of references assisted in having trust in the information provided on the LactaMap website:

Just having references after each section does give you that reassurance that what you are reading is true and relevant. [P6]

One user commented on the publication date of references cited, worried about the relevance of those that were more than 10 years old. This user suggested that a 'last updated' date would help with trust.

Affiliation of the LactaMap website with a university was also positively associated with credibility.

\section{Table 2. Participant details}

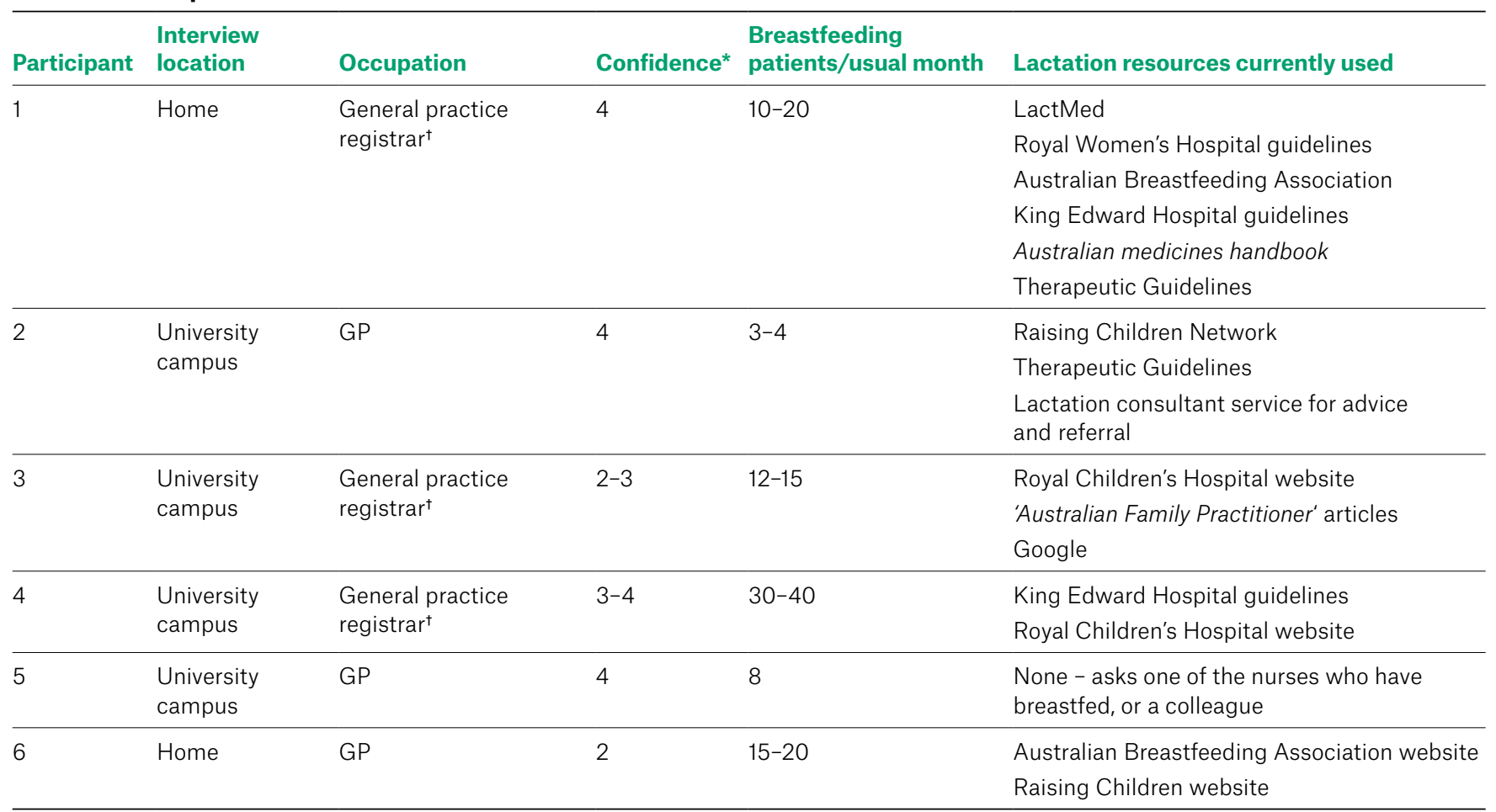

${ }^{*}$ Confidence was rated on a Likert scale from 1 (not confident at all) to 5 (very confident)

${ }^{\dagger} A$ general practice registrar is a medical practitioner who has been accepted into medical specialty training to specialise as a general practitioner. GP, general practitioner 


\section{Desirable}

Desirability considered website design elements that evoked emotion and appreciation. Feedback was very positive:

I think it looks really good. There's nothing like it. Nothing else that I've ever seen like it. [P1]

Users liked the clear layout, colour and text, preferring 'not to have stuff all over the screen' [P6].

\section{Findable}

Findability was focused on whether it was easy to navigate around the website and locate relevant content. Again, most quotes related to an aspect of the website that the user liked. The LactaMap decision tree (care pathway) was well received:

It's like a good invitation like, 'Oh okay, I don't have to know much, I just have to know these four things, and then the system, the computer's going to help me'. And then you can read on, and you're sort of already drawn into it, into the larger part of the description. [P1]

Many of the quotes relating to findability were simply statements that the website was easy to use, easy to navigate and intuitive.

Findability issues ranked as critical or severe were all related to how users could follow their trail back to where they had come from and difficulties in relocating the homepage:

\section{I really like that first page. I'm not sure how to get back there though. [P1]}

\section{Useable \\ This theme related to whether users could effectively and efficiently complete their task, which was to reach a decision endpoint for the case scenario they were provided. The largest number of codes were sorted into this theme. Website features such as photos and videos attracted usability rankings from both ends of the scale. Images were something users liked about the website, but it was also noted that they could be both helpful to show patients or distressing. One user suggested this could be addressed by}

having the option of closing pictures. Videos were considered valuable, but users noted that they were poorly labelled making expected content hard to identify:

That is showing me what? There's no label, is it showing a video of a blocked duct? [P1]

Importantly for website design, five of the six users identified issues with the website registration process. These were clear barriers to getting started and were ranked as critical.

One quote relating to the layout of the decision tree stated that the user would like a way to be able to see the steps they had travelled down the decision tree:

\section{So, it would be good if that was more clear and there was a way to see them all at once. $[\mathrm{P} 1]$}

\section{Useful}

This theme related to whether the LactaMap website had practical value to the user.

Most comments about usefulness related to website content and patient information. Users were pleasantly surprised at the depth of information on the website, which was discovered quickly as they used the website to locate content required to address the case scenario:

The more I used the website, the more I realised that there is a significant amount of information contained in it. [P3]

\section{Wow, it's very comprehensive. [P5]}

They also appreciated the patient information documents that could be printed out or emailed from a 'no-reply' email direct from the website. These provide clinical information summaries in simple language on a range of topics relevant to breastfeeding:

You can send it? Oh, that's really helpful. So you can send it directly to them ... and you can download it. Okay. [P4]

\section{Valuable}

The last theme focused on findings related to whether the user was satisfied with the LactaMap website and believed that it delivered value. This was a subjective assessment that related to the overall website experience. All codes allocated to this theme received a positive ranking:

I think it looks really good. There's nothing like it. Nothing else that I've ever seen like it. [P1]

I'd use it tomorrow. [P2]

I think this is a really, really good website. [P5]

Wow, this is good. [P5]

\section{Discussion}

This study provided data that enabled understanding of the GP user's experience with the intervention prototype. This was a necessary step in the intervention's design to encourage its adoption and use by GPs. ${ }^{13}$ The results indicated that the experience was positive. With a view to further improving the user's experience, the understanding obtained from the analysis of the integrated responses to the Think Aloud and the semi-structured interview was used to adapt the website.

Both the Think Aloud and semi-structured interview contributed data relating to aspects of user experience. Usability ranking of coded data allowed prioritisation of modifications, identified by study participants, that affected task completion. The small number of issues that were ranked as critical or severe showed the methodology's use for website developers to refine the prototype. Examples of refinements included a simplified website registration process, better labelling of videos, a 'home' icon, inclusion of a statement indicating a 'last updated' date on all content pages, the ability to close images (useful if the patient found them distressing; Figure 2) and 'breadcrumbs' to show the user's location in the decision tree (Figure 3).

\section{Limitations of the study}

Increased time pressure in actual clinical situations may unmask LactaMap user interface issues that result in a higher 
degree of user frustration if there is difficulty in task completion. Further studies in a real-world setting would be useful to understand this as well as the effect of changes made to the user interface resulting from this study.

Response bias, particularly users answering in a way considered more socially acceptable, can be a limitation of qualitative studies. This was mitigated by integrating the task-oriented Think Aloud method, which required a higher cognitive load likely to produce honest and less strategic responses, and by encouraging GPs to discuss aspects of the website that they both liked and disliked. ${ }^{31}$ GPs were also informed that their views would provide important input for finalising website design.

\section{Conclusion}

LactaMap is designed to support GPs to provide crucial care for breastfeeding families. This study showed the integration of two complementary qualitative approaches to evaluate the website user experience. Evaluation of LactaMap during development found that the user experience of the LactaMap website was positive and provided important information about factors likely to influence its adoption and use. This guided changes in user interface

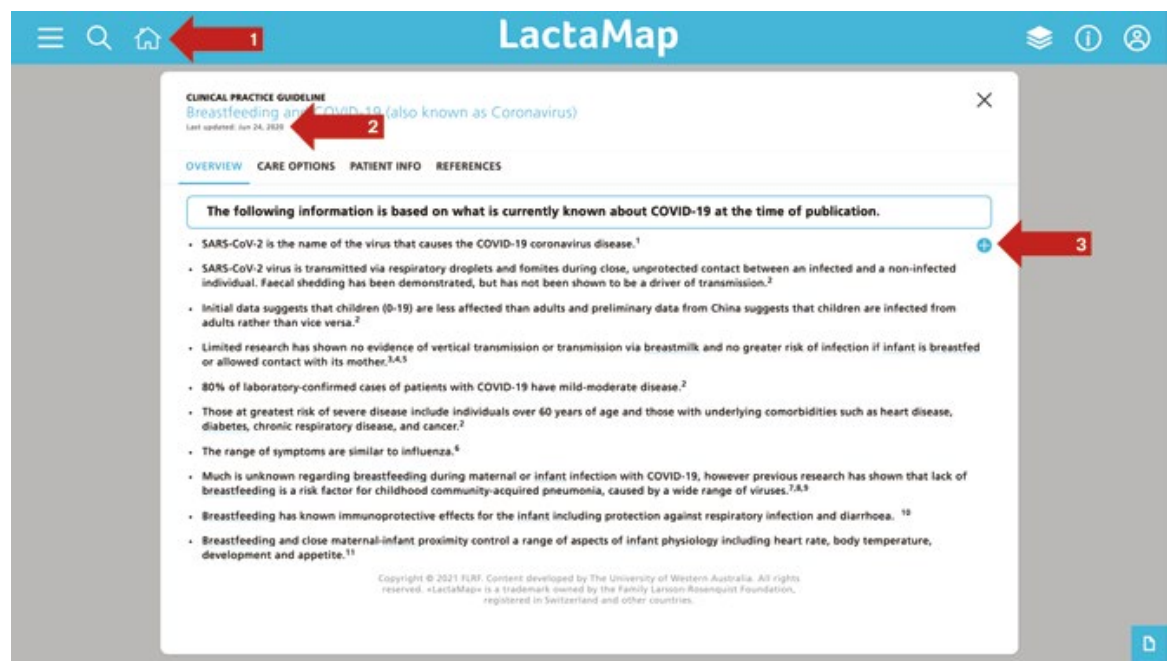

Figure 2. Examples of refinements made to LactaMap website, ${ }^{32}$ indicating: 1) home icon, 2) last updated date, and 3) icon that has been clicked to close image

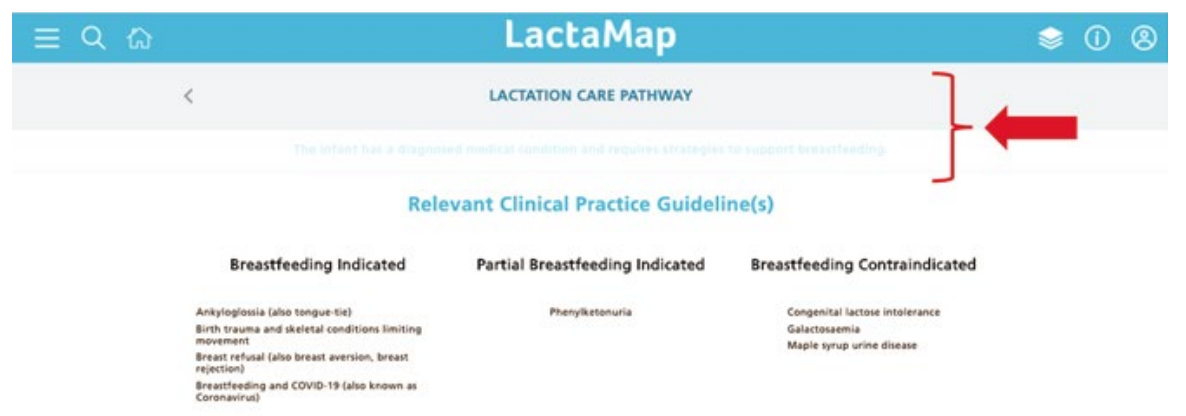

Figure 3. Example of refinement made to LactaMap website showing 'breadcrumbs' that indicate user's location in decision tree ${ }^{32}$

design, customising the lactation decision support tool for the unique requirements of general practice.

\section{Authors}

Melinda Boss BPharm, PhD, Senior Research Fellow, School of Allied Health, Faculty of Health and Medical Sciences, University of Western Australia, Perth, WA

Jennifer Turner BPharm, Cert IV Breastfeeding Ed (Counselling), Research Associate, School of Allied Health, Faculty of Health and Medical Sciences, School of Allied Health, University of Western Australia, Perth, WA

Tracey Hirst BCom, MBL, PhD, Research Fellow, Management and Organisations Department, Business School, University of Western Australia, Perth, WA

Douglas Pritchard BPsych (Hons), MBBS, DA (Lon), FRACGP, PhD, Clinical Associate Professor, Division of General Practice, Faculty of Health and Medical Sciences, University of Western Australia, Perth, WA; General Practitioner, Lockridge Medical Centre, Kiara, WA

Rafael Pérez-Escamilla BS (Chemical Engineering), MS (Food Science), PhD (Nutrition), Professor of Public Health, Department of Social and Behavioural Sciences, Yale School of Public Health, New Haven, CT, USA

Rhonda Clifford BPharm, GradDipHospPharm, PhD, Professor of Pharmacy, Head of School of Allied Health, Faculty of Health and Medical Sciences, University of Western Australia, Perth, WA Competing interests: RPE is the recipient of research funding from the Family Larsson-Rosenquist Foundation that supports research for an unrelated project, Becoming Breastfeeding Friendly. MB, $\mathrm{JT}, \mathrm{TH}$ and DP have received payments from the acknowledged research grant awarded to MB (as chief investigator) by the Family Larsson-Rosenquist Foundation for work relating to the LactaMap project, which includes this research.

Funding: Research funding from the Family LarssonRosenquist Foundation is gratefully acknowledged. $\mathrm{MB}$ is the recipient of an Australian Government Research Training Program Scholarship provided by the Commonwealth Government of Australia. The funders had no input in study design, data collection and analysis or preparation of the manuscript.

Provenance and peer review: Not commissioned, externally peer reviewed.

\section{Correspondence to:}

melinda.boss@uwa.edu.au

\section{References}

1. Victora CG, Bahl R, Barros AJ, et al. Breastfeeding in the 21st century: Epidemiology, mechanisms, and lifelong effect. Lancet 2016;387(10017):475-90. doi: 10.1016/S01406736(15)01024-7.

2. Walters DD, Phan LTH, Mathisen R. The cost of not breastfeeding: Global results from a new tool. Health Policy Plan 2019;34(6):407-17. doi: 10.1093/ heapol/czz050.

3. Gunn J, Lumley J, Young D. Visits to medical practitioners in the first 6 months of life. J Paediatr Child Health 1996;32(2):162-66. doi: 10.1111/j.14401754.1996.tb00915.x.

4. Taveras EM, Li R, Grummer-Strawn L, et al. Opinions and practices of clinicians associated with continuation of exclusive breastfeeding. Pediatrics 2004;113(4):e283-90. doi: 10.1542/ peds.113.4.e283. 
5. Lu MC, Lange L, Slusser W, Hamilton J, Halfon N. Provider encouragement of breastfeeding: Evidence from a national survey. Obstet Gynecol 2001;97(2):290-95. doi: 10.1016/s00297844(00)01116-9.

6. Brodribb W, Fallon A, Jackson C, Hegney D. Breastfeeding and Australian GP registrars - Their knowledge and attitudes. J Hum Lact 2008;24(4):422-30. doi: 10.1177/0890334408323547.

7. Pound CM, Williams K, Grenon R, Aglipay M, Plint AC. Breastfeeding knowledge, confidence, beliefs, and attitudes of Canadian physicians. J Hum Lact 2014;30(3):298-309. doi: 10.1177/0890334414535507.

8. Gonzalez AP, Palmer J, Downes K, Young C. Attitudes and knowledge about breastfeeding among obstetrics and gynecology residents. Obstet Gynecol 2014; 123 Suppl 1: 17S-18S. doi: 10.1097/01.AOG.0000447271.74766.b5

9. Zwolsman S, te Pas E, Hooft L, Wieringa-de Waard M, van Dijk N. Barriers to GPs' use of evidence-based medicine: A systematic review. Br J Gen Pract 2012;62(600):e511-21. doi: 10.3399/bjgp12X652382.

10. Boss M, Pritchard D, Clifford R, et al. P-30. LactaMap: A novel approach to lactation care. Paper presented at 19th International Society for Research in Human Milk and Lactation Conference. Kanagawa, JP: ISRHML Conference, 2018.

11. Australian Digital Health Agency. Australia's national digital health strategy. Canberra, ACT: Australian Government, 2018. Available at https:// conversation.digitalhealth.gov.au/australiasnational-digital-health-strategy [Accessed 12 October 2020].

12. Sim I, Gorman P, Greenes RA, et al. Clinical decision support systems for the practice of evidence-based medicine. J Am Med Inform Assoc 2001;8(6):527-34. doi: 10.1136/ jamia.2001.0080527.

13. Horsky J, Schiff GD, Johnston D, Mercincavage L, Bell D, Middleton B. Interface design principles for usable decision support: A targeted review of best practices for clinical prescribing interventions. J Biomed Inform 2012;45(6):1202-16. doi: 10.1016/j. jbi.2012.09.002.

14. Thirsk LM, Clark AM. Using qualitative research for complex interventions: The contributions of hermeneutics. Intl J Qual Methods 2017;16(1):1609406917721068. doi: 10.1177/1609406917721068

15. Craig P, Dieppe P, Macintyre S, et al. Developing and evaluating complex interventions: The new Medical Research Council guidance. BMJ 2008;337:a1655. doi: 10.1136/bmj.a1655.

16. Eldridge SM, Lancaster GA, Campbell MJ, et al. Defining feasibility and pilot studies in preparation for randomised controlled trials: Development of a conceptual framework. PLoS One 2016;11(3):e0150205. doi: 10.1371/journal. pone.0150205.

17. Anthierens S, Tonkin-Crine S, Douglas E, et al. General practitioners' views on the acceptability and applicability of a web-based intervention to reduce antibiotic prescribing for acute cough in multiple European countries: A qualitative study prior to a randomised trial. BMC Fam Pract 2012;13:101. doi: 10.1186/1471-2296-13-101.

18. Rosenbaum SE, Glenton C, Cracknell J. User experiences of evidence-based online resources for health professionals: User testing of The Cochrane Library. BMC Med Inform Decis Mak 2008;8:34. doi: 10.1186/1472-6947-8-34.
19. Joe J, Chaudhuri S, Le T, Thompson H, Demiris G The use of think-aloud and instant data analysis in evaluation research: Exemplar and lessons learned. J Biomed Inform 2015;56:284-91. doi: 10.1016/j.jbi.2015.06.001.

20. Hertzum M, Hansen KD, Andersen HHK. Scrutinising usability evaluation: Does thinking aloud affect behaviour and mental workload? Behav Inf Technol 2009; 28: 165-81. doi: 10.1080/01449290701773842

21. Van Someren M, Barnard Y, Sandberg, J. The think aloud method: A practical guide to modelling cognitive processes. London, UK: Academic Press, 1994.

22. Dicicco-Bloom B, Crabtree BF. The qualitative research interview. Med Educ 2006;40(4):314-21. doi: 10.1111/j.1365-2929.2006.02418.x.

23. Boss M, Hartmann P, Turner J, Pritchard D, Pérez-Escamilla R, Clifford R. Development of LactaPedia: A lactation glossary for science and medicine. Matern Child Nutr 2020;16(3):e12969. doi: 10.1111/mcn.12969.

24. Cullinane M, Amir LH, Donath SM, et al. Determinants of mastitis in women in the CASTLE study: A cohort study. BMC Fam Pract 2015;16:181. doi: 10.1186/s12875-015-0396-5.

25. Gatti L. Maternal perceptions of insufficient milk supply in breastfeeding. J Nurs Scholarsh 2008;40(4):355-63. doi: 10.1111/j.15475069.2008.00234.x.

26. Boss M, Gardner $H$, Hartmann P. Normal human lactation: Closing the gap. F1000Res 2018;7:F1000 Faculty Rev-801. doi: 10.12688/ f1000research.14452.1.

27. Yang D, Cascella M. Tracheomalacia. 2021 Jul 17. In: StatPearls. Treasure Island, FL: StatPearls Publishing, 2021.

28. Moreville P. User experience design. Scottsville, US: Semantic Studios, 2004. Available at semanticstudios.com/user_experience_design [Accessed $31 \mathrm{Jul}$ 2020].

29. Bond RR, Finlay DD, Nugent CD, Moore G, Guldenring D. A usability evaluation of medical software at an expert conference setting. Comput Methods Programs Biomed 2014;113(1):383-95. doi: 10.1016/j.cmpb.2013.10.006.

30. Saunders B, Sim J, Kingstone T, et al. Saturation in qualitative research: Exploring its conceptualization and operationalization. Qual Quant 2018;52(4):1893-907. doi: 10.1007/s11135017-0574-8.

31. Stodel M. But what will people think?: Getting beyond social desirability bias by increasing cognitive load. Int J Mark Res 2015;57(2):313-22.

32. Boss M, Hartmann P, LactaResearch Group. LactaMap. Frauenfeld, CH: Family LarssonRosenquist Foundation, 2019. Available at www. lactamap.com/home [Accessed 2 October 2020]. 